\title{
Analyzing refractive index changes and differential bending in microcantilever arrays
}

\author{
François Huber, ${ }^{1, a)}$ Hans Peter Lang, ${ }^{1}$ Martin Hegner, ${ }^{2}$ Michel Despont, ${ }^{3}$ Ute Drechsler, ${ }^{3}$ \\ and Christoph Gerber ${ }^{1}$ \\ ${ }^{1}$ NCCR Nanoscale Science, Institute of Physics, University of Basel, Klingelbergstrasse 82, \\ CH-4056 Basel, Switzerland \\ ${ }^{2}$ CRANN Trinity College Dublin, College Green, Dublin 2, Ireland \\ ${ }^{3}$ IBM Research GmbH, Zürich Research Laboratory, Säumerstrasse 4, 8803 Rüschlikon, Switzerland
}

(Received 4 July 2008; accepted 21 July 2008; published online 20 August 2008)

\begin{abstract}
A new microcantilever array design is investigated comprising eight flexible microcantilevers introducing two solid bars, enabling to subtract contributions from differences in refractive index in an optical laser read out system. Changes in the refractive index do not contribute undesirably to bending signals at picomolar to micromolar DNA or protein concentrations. However, measurements of samples with high salt concentrations or serum are affected, requiring corrections for refractive index artifacts. Moreover, to obtain a deeper understanding of molecular stress formation, the differential curvature of cantilevers is analyzed by positioning the laser spots along the surface of the levers during $p \mathrm{H}$ experiments. (c) 2008 American Institute of Physics.
\end{abstract}

[DOI: 10.1063/1.2969912]

Recently considerable advancements in the application of microcantilever array based technologies have been achieved in the areas of genomic analysis where single gene products were detected in an unspecific background, shining light on the regulatory processes when and where genes are expressed. ${ }^{1}$ The 200-fold improvement of the sensitivity in the analysis of antibody/antigen interaction with nanomechanical sensors sets the technology on par with surface plasmon resonance acquiring data in the lower nanomolar concentration range. ${ }^{2}$ Moreover analyzing protein DNA binding in a combined assay gives rise to a new device in genomic and proteomic analytics. ${ }^{3}$ These measurements rely on the creation of an interfacial surface stress upon binding of an analyte to probe molecules tethered to a gold coated microcantilever surface. The effect of these molecular interactions on a surface is microcantilever bending in the nanometer range, measured using a laser beam and analyzed with a position sensitive detector. However, the path of the deflected light depends on the optical density of the medium it enters and will change if a medium with a different refractive index is injected. Thus, an artificial change in the deflection is observed. To provide an in-built reference to be able to compensate for such an artificial change in deflection, a new microcantilever array design is introduced here (Fig. 1). It contains in addition to the eight thin microcantilevers two solid bars at each end of the array. Due to their thickness (450 $\mu \mathrm{m})$, the bars will not respond to surface stress changes and therefore provide good indicators for environmental changes such as refractive index or temperature variations. We found that refractive index changes occur during the injection of high salt concentrations, fetal calf serum (FCS) and different concentrations of 1-dodecanethiol but no mea-

\footnotetext{
${ }^{a)}$ Author to whom correspondence should be addressed. Electronic mail:
} francois.huber@unibas.ch. surable effect is observed upon injection of oligonucleotides or proteins up to $10 \mu \mathrm{M}$. We furthermore investigated the bending profile of the microcantilevers in this new design in a liquid environment. For that purpose, the laser beam was positioned at intervals of roughly $50 \mu \mathrm{m}$ along the microcantilever from the hinge to the tip, and the nanomechanical deflection was evaluated to reveal the shape of the differential microcantilever bending profile, resulting in 12 data points along a microcantilever. This finding is an improvement in lateral resolution in a liquid environment of more than twofold compared to previous work, ${ }^{4}$ where the absolute bending of only one microcantilever was analyzed. It was shown empirically and theoretically ${ }^{5}$ that the shape is close to an arc of a circle as also known from the work by Stoney. ${ }^{6}$ However, Jeon et al. ${ }^{7}$ showed that for the formation of self-assembled monolayers on microcantilever surfaces the bending curvature can deviate from a circular shape. Nevertheless, we were able to show that in $\mathrm{pH}$ measurements also the differential bending along the length of the evaluated microcantilevers, i.e., the difference in observed bending of a microcantilever relative to the solid bar follows in a first approximation a circular path.

Most biological experiments require an aqueous environment. For an optical readout of microcantilever bending, as used in many applications ${ }^{8,9}$ this can cause artificial deflections due to changes in the refractive index upon injection of different solutions. Figure 2(a) shows this effect clearly on the solid bar, where an artificial bending signal was created upon serial injections of solutions with increasing salt concentrations. The array was first equilibrated in a $10 \mathrm{mM}$ sodium citrate buffer containing $100 \mathrm{nM} \mathrm{NaCl}$ (SSC buffer). Subsequently, SSC solutions with higher $\mathrm{NaCl}$ concentrations were injected. It looks like there is a bending of up to $600 \mathrm{~nm}$ at a concentration of $1 M \mathrm{NaCl}$. These artificial bending signals increase linearly with the $\mathrm{NaCl}$ concentration. The solid bar with a thickness of $450 \mu \mathrm{m}$, which is 25.000 


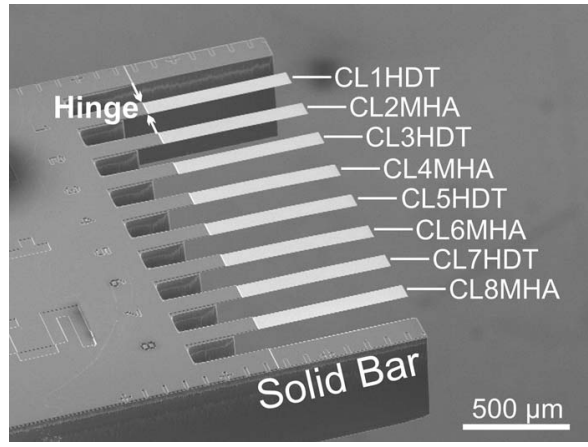

FIG. 1. SEM image of a microfabricated microcantilever array. A microcantilever is $700 \mu \mathrm{m}$ long from the hinge (indicated by two arrows) to the apex, $100 \mu \mathrm{m}$ wide and $1 \mu \mathrm{m}$ thick. The distance from the middle of one microcantilever to the middle of the next one is $250 \mu \mathrm{m}$. The microcantilevers were coated with $2 \mathrm{~nm}$ of $\mathrm{Ti}$ and $20 \mathrm{~nm}$ of Au on top. The Au layer served as a substrate to functionalize the microcantilevers with MHA or HDT. Here seven of the eight microcantilevers (microcantilevers 2-8) and the solid bar were used to analyze bending and refractive index changes.

times stiffer as compared to the $1 \mu \mathrm{m}$ thick microcantilevers, cannot bend due to prevailing surface stress measured with the beam deflection method. However an apparent bending is observed, indicating an artificial effect due to changes in the refractive index of the injected liquid. For comparison the solutions were measured using a refractometer (ALV differential refractometer, ALV Laservertriebsgesellschaft $\mathrm{mbH}$ ). As shown in Fig. 2(b) changes in $n^{\prime} / n$, the ratio between the refractive indices of the measured $\mathrm{NaCl}$ concentration and a reference are in the range between $9 \times 10^{-4}$ and $9 \times 10^{-3}$ showing a linear increase with the $\mathrm{NaCl}$ concentration. Contrary to these findings are the measurements presented in Fig. 2(c) with protein solutions and additionally with single strand oligonucleotides (data not shown). Here bovine serum albumin (BSA), and 12-mer oligonucleotide BioB2 (Ref. 9) solutions were used. BSA was dissolved in $10 \mathrm{mM}$ phosphate buffer containing $2.7 \mathrm{mM} \mathrm{KCl}$ and $137 \mathrm{mM} \mathrm{NaCl}$ (PBS) and the oligonucleotides in SSC buffer. Here no artificial signal is observed with concentrations commonly used in recent work, ${ }^{1-3}$ which shows that microcantilever measurements in that concentration range are not affected. Again measurements with a refractometer were carried out, shown in Fig. 2(d) for BSA solutions applied to the microcantilevers and for ssDNA (data not shown) for comparison with the $\mathrm{NaCl}$ measurements. While the increase in BSA concentrations from 2 to $10 \mu \mathrm{M}$ is observed in the refractometer measurements, no signal is obtained with the solid bar. This is due to the small increase in $n^{\prime} / n$ from $2 \times 10^{-5}$ to $8 \times 10^{-5}$ as compared to the $\mathrm{NaCl}$ data which are 10-100 times larger. Similarly, no signal can be observed with the solid bar using oligonucleotide concentrations ranging from $1 \mathrm{nM}$ to $10 \mu \mathrm{M}$ (data not shown). Again the reference measurements with the refractometer show a 1000 -fold lower refractive index change than observed in the $\mathrm{NaCl}$ experiment. Figure 2(e) shows an experiment where FCS in PBS is injected in a concentration range from $1 \%$ to $100 \%$. We can see a change in refractive index as the concentration increases as well as an increase in signal noise. The last observation can be explained by the complex composition of serum, which contains proteins and aggregates that either adsorb quickly to surfaces or disturb the path of the laser beam. These two observations are important for biological experiments where the addition of serum may be necessary to stabilize proteins or if direct experiments without further purification of blood samples are required. The last refractive index experiment presented in Fig. 2(f) analyzes the effects of various 1-dodecanthiol concentrations in ethanol. Significant artificial signals can be observed at 10,5 , and $2 \mathrm{mg} / \mathrm{ml}$ 1-dodecanethiol; lower concentrations do not have an influence on the refractive index.
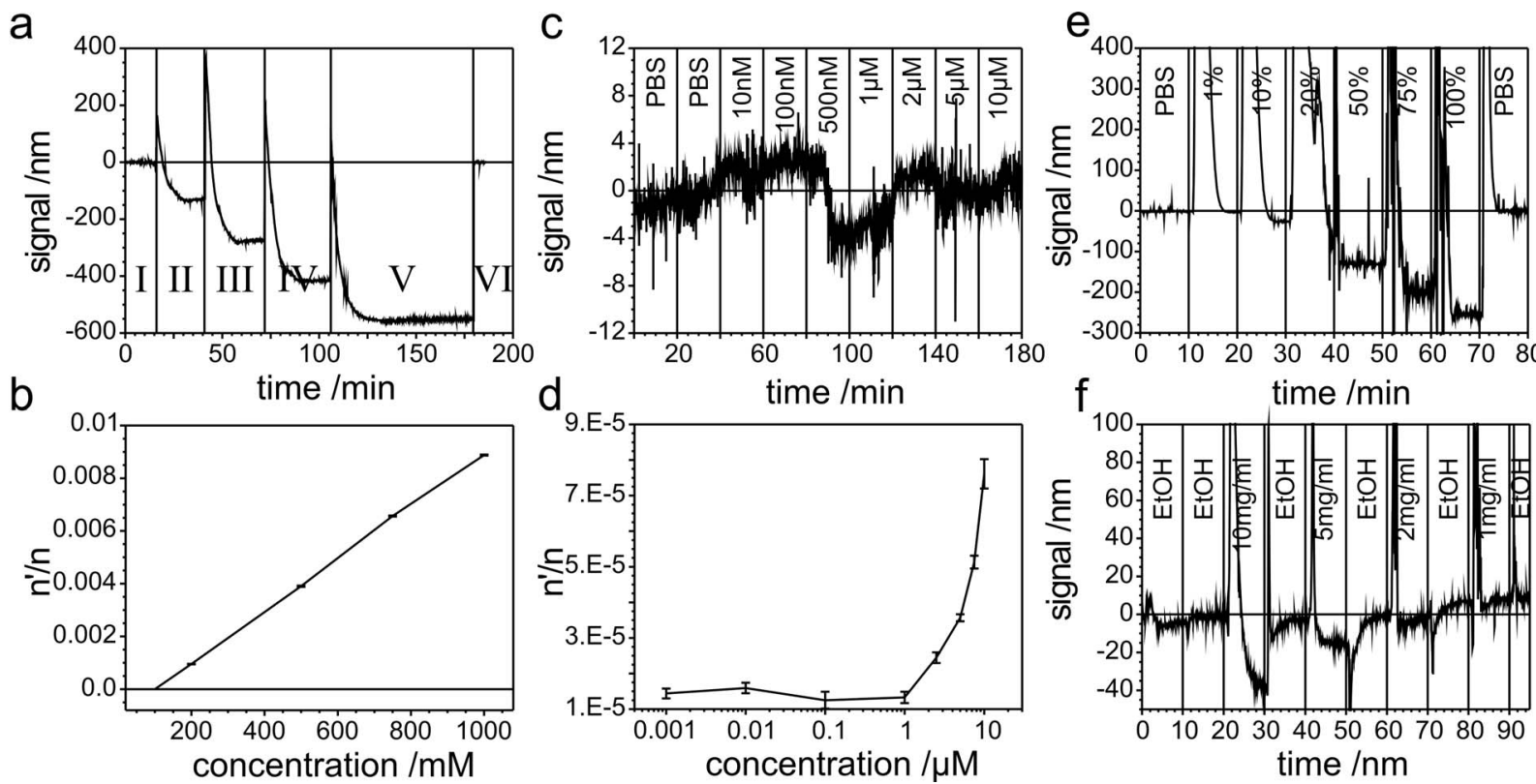

FIG. 2. Refractive index changes due to the injection of different solutions monitored with the solid bar. (a) Injection of SSC solutions containing different amounts of $\mathrm{NaCl}, 100 \mathrm{mM}$ (I), $200 \mathrm{mM}$ (II), $500 \mathrm{mM}$ (III), $750 \mathrm{mM}$ (IV) to $1000 \mathrm{mM}$ (V), and $100 \mathrm{mM}$ (VI) again. (c) PBS solutions containing $10 \mathrm{nM}$ to $10 \mu \mathrm{M}$ BSA were injected. (e) PBS solutions containing $1 \%$ up to $100 \%$ FCS were injected. (f) Injection of different concentrations of 1-dodecanethiol in ethanol ranging from 10 down to $1 \mathrm{mg} / \mathrm{ml}$. (b) and (d) show corresponding refractive index measurements of the salt and protein solutions carried out with a refractometer. 


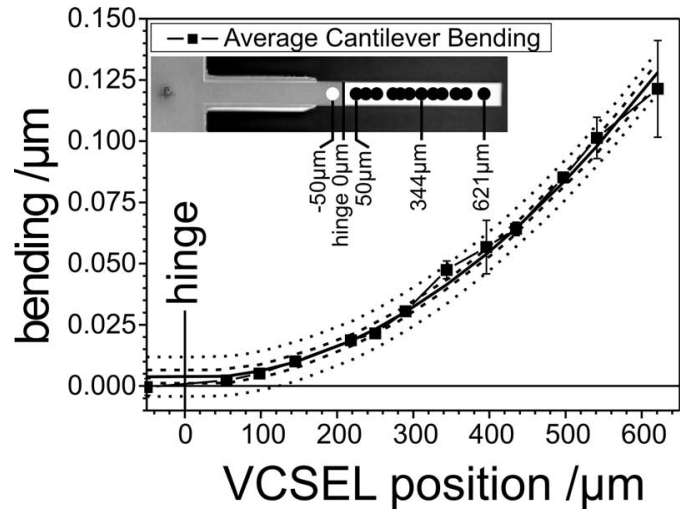

FIG. 3. Analysis of the differential bending of the microcantilevers upon $\mathrm{pH}$ changes. The thick black line shows the circular model $\left[y=b-\sqrt{\left(r^{2}-x^{2}\right)}\right]$ used to fit the differential deflection data (black squares), the thin dotted lines below and above the fit show the 95\% confidence interval and the dashed line above and below indicate the $95 \%$ prediction interval. An $R^{2}$ $=0.993$ and a bending radius of $1.541 \pm 0.047 \mathrm{~m}$ for the differential bending was calculated. For each data point, three measurements were averaged and the standard deviation was plotted. The stars correspond to the laser spots indicated in the inset. The inset shows the different positions of the laser spots from the vertical cavity surface emitting lasers (VCSELs) along a microcantilever. The uneven distribution is due to manual positioning along the microcantilever. The spot size is about $50 \mu \mathrm{m}$ in diameter.

For the following experiments presented in Fig. 3, microcantilevers were functionalized, as shown in Fig. 1, with either hexadecanethiol (HDT) or mercaptohexadecanoic acid (MHA). Because of its acidic head group, MHA responds to changes in $p \mathrm{H}^{10,11}$. The $p \mathrm{H}$ experiments were extended to assess the differential bending of the microcantilevers from the new array design. Differential readings, HDT data were subtracted from MHA data, were acquired and averaged for each position indicated in the inset of Fig. 3. For the analysis a simple Eq. (1) describing a circle was chosen to fit the data.

$$
y=b-\sqrt{\left(r^{2}-x^{2}\right)},
$$

where $y$ is the bending of the microcantilever in nanometers, $b$ is an offset, $r$ is the bending radius, and $x$ depicts the position of the laser spot along the microcantilever. A differential bending radius of $1.541 \pm 0.047 \mathrm{~m}$ was calculated. With the help of Stoney's equation and Sader's correction ${ }^{5}$ [Eq. (2)] a differential surface stress $(\Delta \sigma)$ can be calculated

$$
\Delta \sigma=\frac{s \Delta z E T^{2}}{3 L^{2}(1-\nu)},
$$

with $s=0.83$ (Sader's correction), $\Delta z=121 \mathrm{~nm}$ equalling the deflection at a distance of $621 \mu \mathrm{m}$ from the hinge and being the last point measured, $E=1.2 \times 10^{11} \mathrm{~Pa}$ (Young's modulus of silicon), $T=10^{-6} \mathrm{~m}$ is the microcantilever thickness and the poisson ratio $\nu=0.25$, a differential surface stress of $\Delta \sigma=1.39 \times 10^{-2} \mathrm{~N} / \mathrm{m}$ can be derived. The results indicate that any nonspecific or specific effect, that are capable of producing a surface stress, will bend microcantilevers in a circular way as observed with the HDT modified or nonpassivated interfaces (data not shown).

This work shows the importance of differential measurements with microcantilevers and of an array-type configuration. While changes in the refractive index are negligible at concentrations commonly used in protein and oligonucleotide experiments in homogeneous samples, this is no longer true if different $\mathrm{NaCl}$ concentrations, FCS containing buffers or ethanol with various concentrations of 1-dodecanethiol are used in experiments. Here a significant artificial bending signal is observed. Refractive index changes may explain anomalous bending behavior of microcantilevers reported elsewhere ${ }^{7}$ in single cantilever measurements. It was also validated that the new microcantilever array design behaved in the same way as shown by others for the older design ${ }^{10,1}$ without the solid bars. The new microcantilever array design with solid bars will clearly help in assessing changes in the refractive index and its influence in experiments with samples of a complex composition (e.g., biological serum).

${ }^{1}$ J. Zhang, H. P. Lang, F. Huber, A. Bietsch, W. Grange, C. Certa, R. McKendry, H.-J. Güntherodt, M. Hegner, and C. Gerber, Nat. Nanotechnol. 1, 214 (2006)

${ }^{2}$ N. Backmann, C. Zahnd, F. Huber, A. Bietsch, A. Plückthun, H. P. Lang, H.-J. Güntherodt, M. Hegner, and C. Gerber, Proc. Natl. Acad. Sci. U.S.A. 102, 14587 (2005).

${ }^{3}$ F. Huber, M. Hegner, C. Gerber, H.-J. Güntherodt, and H. P. Lang, Biosens. Bioelectron. 21, 1599 (2006).

${ }^{4}$ S. Jeon and T. Thundat, Appl. Phys. Lett. 85, 1083 (2004).

${ }^{5}$ J. E. Sader, J. Appl. Phys. 89, 2911 (2001).

${ }^{6}$ G. G. Stoney, Proc. R. Soc. London, Ser. A 82, 172 (1909).

${ }^{7}$ S. Jeon, N. Jung, and T. Thundat, Sens. Actuators B 122, 365 (2007).

${ }^{8}$ J. Fritz, M. K. Baller, H. P. Lang, H. Rothuizen, P. Vettiger, E. Meyer, H.-J. Güntherodt, C. Gerber, and J. K. Gimzewski, Science 288, 316 (2000).

${ }^{9}$ R. McKendry, J. Zhang, Y. Arntz, T. Strunz, M. Hegner, H. P. Lang, M. K. Baller, U. Certa, E. Meyer, H.-J. Güntherodt, and C. Gerber, Proc. Natl. Acad. Sci. U.S.A. 99, 9783 (2002).

${ }^{10}$ J. Fritz, M. K. Baller, H. P. Lang, T. Strunz, E. Meyer, H.-J. Güntherodt, E. Delamarche, C. Gerber, and J. K. Gimzewski, Langmuir 16, 9694 (2000).

${ }^{11}$ M. Watari, J. Galbraith, H. P. Lang, M. Sousa, M. Hegner, C. Gerber, M. A. Horton, and R. A. McKendry, J. Am. Chem. Soc. 129, 601 (2007). 